\title{
Induction- and conditioning-protocol dependent involvement of NR2B-containing NMDA receptors in synaptic potentiation and contextual fear memory in the hippocampal CAI region of rats
} Xue-Han Zhang1,2, Long-Jun Wu², Bo Gong ${ }^{2}$, Ming Ren², Bao-Ming Li*1 and Min Zhuo*1,2

Address: ${ }^{1}$ Institute of Neurobiology and State Key Laboratory of Medical Neurobiology, Institutes of Brain Science, Fudan University, Shanghai 200032, PR China and 2Department of Physiology, Faculty of Medicine, University of Toronto, 1 King's College Circle, Toronto, Ontario M5S 1A8, Canada

Email: Xue-Han Zhang - xuehan.zhang@utoronto.ca; Long-JunWu - longjun.wu@utoronto.ca; Bo Gong - bo.gong@utoronto.ca; Ming Ren - ming.ren@utoronto.ca; Bao-Ming Li* - bmli@fudan.edu.cn; Min Zhuo* - min.zhuo@utoronto.ca

* Corresponding authors

Published: 30 September 2008

Molecular Brain 2008, 1:9 doi:10.1 186/1756-6606-1-9
Received: 26 August 2008

Accepted: 30 September 2008

This article is available from: http://www.molecularbrain.com/content/I/I/9

(C) 2008 Zhang et al; licensee BioMed Central Ltd.

This is an Open Access article distributed under the terms of the Creative Commons Attribution License (http://creativecommons.org/licenses/by/2.0), which permits unrestricted use, distribution, and reproduction in any medium, provided the original work is properly cited.

\begin{abstract}
Long-term potentiation (LTP) in the hippocampal CAI region requires the activation of $\mathrm{N}$-methylD-aspartate receptors (NMDARs). Studies using genetic and pharmacological approaches have reported inconsistent results of the requirement of NR2B-containing NMDARs in LTP in the CAI region. Pharmacological studies showed that NR2B-containing NMDARs are not required for LTP, while genetic studies reported that over-expression of NR2B-NMDARs enhances LTP and hippocampus-dependent memory. Here, we provide evidence showing that the functional role of NR2B-NMDARs in hippocampal LTP and memory depends on LTP-inducing and behaviorconditioning protocols. Inhibition of NR2B-NMDARs with the NR2B selective antagonist ifenprodil or Ro25-698I suppressed LTP induced by spike-timing protocol, with no impact on LTP induced by pairing protocol or two-train high-frequency stimulation (HFS) protocol. Inhibition of NR2BNMDARs did not affect the late phase LTP induced by four-train HFS. $\mathrm{Ca}^{2+}$ imaging showed that there was difference in kinetics of intracellular $\mathrm{Ca}^{2+}$ signals induced by spiking-timing and pairing protocols. Pre-training intra-CAI infusion of ifenprodil or Ro25-698I impaired the contextual fear memory induced by five CS-US pairings, with no effect on the memory induced by one CS-US pairing.
\end{abstract}

\section{Introduction}

N-methyl-D-aspartate receptors (NMDARs) are critical for synaptic plasticity in the hippocampus and hippocampusdependent learning and memory [1-3]. NMDAR is heteromer. Functional NMDAR has a combination of NR1 subunit and at least one of NR2 subunits (A-D). In the hippocampus, NR2A- and NR2B are two predominant NR2 subunits for NMDARs $[4,5]$. The NR2-subunit com- positions of NMDAR, whether they are NR2A- or NR2Bcontaining, determines the pharmacological and kinetic properties of the NMDAR-mediated currents [6-8]. Studies have suggested that NR2A- vs. NR2B-NMDARs may mediate distinct synaptic functions $[9,10]$.

Synaptic long-term potentiation (LTP) has been widely accepted as a synaptic mechanism for learning, 
memory and other higher-order brain functions $[1,2]$. LTP can be induced in brain-slice preparations by different induction protocols $[1,11]$. A recent study by Wang's group reported that NR2A- and NR2BNMDARs may be differentially involved in synaptic plasticity in the CA1 region, with LTP being mediated by NR2A-NMDAR and LTD by NR2B-NMDAR [9]. However, the conclusion that NR2B-NMDAR is selectively involved in the induction of LTD but not LTP has been challenged by the studies using NR2A-knockout mice $[12,13]$ and over-expression of NR2BNMDARs [14], suggesting the existence of NR2A-independent forms of LTP. Furthermore, it should be noted that these previous studies used different slice preparations and, especially, different protocols to induce LTP. It might be possible that NR2B-NMDARs contribute differentially to LTP induced under different conditions.

Hippocampus is an essential structure for flexible spatial memory and contextual fear memory. For example, inactivation of or lesion to the hippocampal CA1 region results in a severe deficit in contextual fear memory $[15,16]$. It is well known that NMDARs in the CA1 region are involved not only in memory acquisition $[3,17,18]$, but in memory retrieval as well [19]. These previous studies used non-selective NMDAR antagonists like MK-801 to access the contributions of hippocamopal NMDARs to memory acquisition and retrieval. The role of NR2BNMDAR has been largely unclear and remains to be established.

In the present study, we examined the effects of selective blockade of NR2B-NMDARs on LTP induced by different protocols including spike-timing protocol $[20,21]$, paring protocol and high-frequency stimulation (HFS) protocol. We also investigated the intracellular calcium signals under different LTP-inducting protocols. We further addressed the impacts of intra-CA1 blockade of NR2BNMDARs on the acquisition and retrieval of contextual fear memory induced by different conditioning strengths.

\section{Materials and methods Animals}

Rats and mice were used for the present study. The animals housed in plastic cages and maintained on a 12-h light/dark cycle. Food and water were available ad libitum throughout the experiment. All experimental procedures were in accordance with the Guide for the Care and Use of Laboratory Animals issued by the National Institutes of Health, USA (1996) and approved by the Ethical Committee of Animal Experiments at Fudan University Institute of Neurobiology (Shanghai, China) and by the Animal Studies Committee at the University of Toronto (Toronto, Canada).

\section{Drugs}

To address the functions of NR2B-containing NMDARs, we used two selective antagonists: the non-competitive NR2B antagonist ifenprodil tartrate salt (Sigma-Aldrich Co., USA) and its derivative Ro25-6981 hydrochloride (Tocris, UK). In LTP recording experiments, we used the 3 $\mu \mathrm{M}$ dose of ifenprodil and the $0.3,0.5$ or $3.0 \mu \mathrm{M}$ doses of Ro25-6981 to block NR2B-NMDARs. In behavioral experiments, we locally infused the $0.2 \mu \mathrm{g}$ dose of ifenprodil or the $5.0 \mu \mathrm{g}$ dose of Ro25-6981 into the CA1 region to inhibit NR2B-NMDARs.

To dissect the functions of NR2A-containing NMDARs, the relatively selective NR2A antagonist NVP-AAM077 (a generous gift from Novartis Pharma, Basel, Switzerland) was used. We selected the $0.3 \mu \mathrm{M}$ dose of NVP-AAM077 in LTP recording experiments to dissect NR2A-NMDAR contributions, as our previous study showed that NVPAAM077 at the dose of $0.3 \mu \mathrm{M}$ did not affect NR2BNMDAR currents in vitro [22]. For behavioral experiments, we locally infused the 0.012 and $0.12 \mu \mathrm{g}$ doses of NVP-AAM077 into the CA1 region to block NR2ANMDARs. The $0.12 \mu \mathrm{g} / \mu \mathrm{l}$ dose of NVP-AAM077 is equal to the $0.2 \mu \mathrm{g} / \mu \mathrm{l}$ dose of ifenprodil in molarity.

\section{Whole-cell patch clamp recordings}

Sprague-Dawley rats (male, 200-220 g, 8-10 week old) were used for the experiments of whole-cell patch clamp recordings. Rats were anesthetized with inhaled isoflurane. Transverse hippocampal slices (300 $\mu \mathrm{m}$ in thickness) were rapidly prepared. Slices were incubated at room temperature for at least $1 \mathrm{~h}$, and were then transferred to a recording chamber. The chamber was perfused at a speed of $2 \mathrm{ml} / \mathrm{min}$ with artificial cerebrospinal fluid (ACSF) consisting of (in $\mathrm{mM}$ ): $124 \mathrm{NaCl}, 2.5 \mathrm{KCl}, 2$ $\mathrm{CaCl}_{2}, 1 \mathrm{MgSO}_{4}, 25 \mathrm{NaHCO}_{3}, 1 \mathrm{NaH} 2 \mathrm{PO}_{4}$, and 10 glucose (saturated with $95 \% \mathrm{O}_{2}$ and $5 \% \mathrm{CO}_{2}$ ) and $100 \mu \mathrm{M}$ picrotoxin. The $\mathrm{CA} 3$ region was removed in order to reduce transmission of picrotoxin-induced epileptiform bursting to the $\mathrm{CA} 1$ region. Whole-cell patch clamp recordings were made in CA1 pyramidal neurons. Targeted neurons were voltage clamped at $-60 \mathrm{mV}$. EPSCs were evoked by extracellular stimulation of Schaffer collaterals using a bipolar stimulating electrode at a rate of $0.02 \mathrm{~Hz}$. The stimulating electrode was placed at about $100 \mu \mathrm{m}$ distant from the cell body.

Spike-timing and pairing protocols were used to induce LTP in patched cells. In spike-timing protocol, three presynaptic stimuli at $30 \mathrm{~Hz}$, which caused three EPSPs, were paired with three postsynaptic action potentials (APs), and did so 15 times with an interval of 5 s. Presynaptic stimulus was delivered $10 \mathrm{~ms}$ before postsynaptic AP. The recording pipette (3-5 M $\Omega$ ) was filled with solution containing (in mM): $145 \mathrm{~K}$-gluconate, $5 \mathrm{NaCl}, 1 \mathrm{MgCl}_{2}, 0.2$ 
EGTA, 10 HEPES, $2 \mathrm{Mg}$-ATP, and $0.1 \mathrm{Na}_{3}$-GTP (adjusted to $\mathrm{pH} 7.2$ with $\mathrm{KOH}$ ). In pairing protocol, a train of 200 stimulation pulses (at $2 \mathrm{~Hz}$ ) were delivered presynaptically, paired with a depolarization (to $-5.0 \mathrm{mV}$ ) of postsynaptic cell (patched cell). The recording pipette (3-5 M $\Omega$ ) was filled with solution containing (in mM): 102 Cs-gluconate, $3.7 \mathrm{NaCl}, 11$ BAPTA, 0.2 EGTA, 20 HEPES, $2 \mathrm{Mg}$ ATP, 0.3 Na3-GTP and 5 QX-314 (adjusted to pH 7.2 with $\mathrm{CsOH}$ ). After EPSC recording was stable for $10 \mathrm{~min}$, spiketiming protocol or pairing protocol was used to induce LTP. The access resistance was $15-30 \mathrm{M} \Omega$. The input resistance was monitored throughout the recording experiment. Data were discarded if input resistance changed by more than $20 \%$.

\section{Field potential recordings}

C57BL/6 mice (male, 6-8 week old) were used for the experiments of field-potential recordings. Mice were anesthetized with inhaled isoflurane. Transverse slices of hippocampus were rapidly prepared and maintained in an interface chamber at $28^{\circ} \mathrm{C}$, in which the slices were perfused with ACSF. The slices were kept in the recording chamber for at least $2 \mathrm{~h}$ before the experiments. A bipolar tungsten stimulating electrode was placed in the stratum radiatum of the CA1 region, and extracellular field potentials were recorded in the stratum radiatum, using a glass microelectrode (3-12 M $\Omega$, filled with ACSF). Stimulation intensity was adjusted to produce a response with $0.5 \sim 1.0$ $\mathrm{mV}$ amplitude. Test responses were elicited at $0.02 \mathrm{~Hz}$. Two trains of HFS (100 pulses at $100 \mathrm{~Hz}$, with inter-train interval of $20 \mathrm{~s}$ ) were used to induce early LTP (E-LTP) and four trains of HFS (100 pulses at $100 \mathrm{~Hz}$, with inter-train interval of $5 \mathrm{~min}$ ) to induce late LTP (L-LTP).

\section{Calcium Imaging}

C57BL/6 mice (male, 3-4 week old) were used for the experiments of calcium imaging. Mice were anesthetized with inhaled isoflurane and were decapitated. Transverse slices of hippocampus (300 $\mu \mathrm{m}$ in thickness) were prepared and transferred to a submerged recovery chamber with oxygenated $\left(95 \% \mathrm{O}_{2}\right.$ and $\left.5 \% \mathrm{CO}_{2}\right)$ ACSF at room temperature. Oregon green BAPTA-1 (OGB-1, 0.4 mM; Molecular Probes) was dialyzed into hippocampal CA1 neurons by whole-cell patch pipettes. Once patched in whole-cell mode, the neurons were maintained for at least 10 min to allow for filling with OGB-1 before image acquisition. Fluorescent signals were imaged by a confocal microscope (Fluoview FV 1000, Olympus, Tokyo, Japan). The laser with a wavelength of $488 \mathrm{~nm}$ was used for excitation and fluorescence was recorded through a bandpass filter (500-550 nm). The images were acquired using a $40 \times, 0.8$ numeric aperture water-immersion objectives every $0.5 \mathrm{~s}$ after a 0.188 -s exposure to $488-\mathrm{nm}$ light. XYT image galleries were collected. Average fluorescence intensity in the region of interests (ROI) was measured for quantification. The ROI was around $100 \mu \mathrm{m}$ from soma.
The intensity was expressed as F/F0, where F0 is the fluorescence intensity before LTP induction.

\section{Fear conditioning}

Fear conditioning was performed in a plexiglas conditioning chamber with a metal grid floor (San Diego Instruments, USA). Infrared equipment was located on the walls to monitor freezing behavior of rats. Rats were given 5 min to acclimate to the chamber pre-conditioning. Two conditioning protocols were employed. For one CS-US pairing protocol, rats were presented with one tone (conditioned stimulus, CS; $2.2 \mathrm{kHz}$ and $96 \mathrm{~dB}$ for $30 \mathrm{~s}$ ), which co-terminated with a foot shock (unconditioned stimulus, US; $1.0 \mathrm{~mA}, 2 \mathrm{~s}$ ). For five CS-US pairing protocol, rats received five CS-US pairings ( $1.0 \mathrm{~mA}, 0.5 \mathrm{~s}$ for each US), with inter-pairing interval of 90-120 s [23]. After conditioning, rats were placed back to home cages.

Memory retention was tested $48 \mathrm{~h}$ post-conditioning. For testing of contextual fear memory, rats were placed into the original chamber, where the rats had been conditioned, and allowed to stay there for $3 \mathrm{~min}$ without tone or footshock. Freezing response during this period was used as a measure for contextual fear memory. For testing of auditory fear memory, rats were placed into a novel chamber for $90 \mathrm{~s}$ and were then given three CSs, each lasting $30 \mathrm{~s}$ with inter-CS interval of $20 \mathrm{~s}$. Freezing response during the CS presentations was used as a measure for auditory fear memory.

\section{Histology}

To verify the locations of drug infusion, rats were anesthetized with pentobarbital sodium (50 mg/kg, i.p.) and perfused transcardially with saline, followed by $4 \%$ (vol/vol) formaldehyde solution. Rat brains were placed into 30\% (wt/vol) sucrose solution and subsequently cut into 40 50 $\mu \mathrm{m}$ sections with a cryostat (Leica CM900, Germany). Brain sections were mounted on gelatin-subbed glass slides and stained with neural red (1\% in ddH2O). Images were taken using a light microscope (Leica DMRXA Q5001W) equipped with a CCD camera.

\section{Data analysis}

Data in the text and figures are expressed as means \pm SEM. Student's t-test was used to compare LTP data. An one-way Analysis of Variance (ANOVA) was employed to compare behavioral data, with planned comparisons as post hoc analysis. In all cases, $\mathrm{p}<0.05$ was considered significant. Statistical analysis was performed using STATISTICA (StatSoft Inc., Chicago, IL, USA).

\section{Results LTP induced by spike-timing protocol}

To test whether the involvement of NR2B-NMDARs is dependent on specific LTP induction paradigm, we first examined the role of NR2B- and NR2A-NMDARs in LTP 
induced by spike-timing protocol (also named EPSPs-APs protocol) (Figure 1A). To be consistent with the experimental conditions used by Wang's Group [9], we first performed whole-cell patch clamp recordings in CA1 pyramidal neurons from rat hippocampus. Spike-timing protocol caused a significant potentiation of synaptic responses (Figure 1B: $190.8 \pm 12.2 \%$ of baseline at $25-30$ min post-induction, $\mathrm{n}=8$ slices; $p<0.05$ vs. baseline). We then tested the possible contribution of NR2B-NMDARs. The non-competitive, selective NR2B-NMDAR antagonist ifenprodil $(3 \mu \mathrm{M})$ was perfused throughout the experiments. As shown in Figure 1C and 1E, ifenprodil significantly reduced the potentiation $(121.4 \pm 10.7 \%$ of baseline, $\mathrm{n}=8$ slices, $p<0.05$ vs. control). Similar inhibitory effects were found with another NR2B-NMDAR antagonist Ro25-6981 (Figure 1D and 1E: For $0.3 \mu \mathrm{M}$ Ro25-6981, $130.6 \pm 13.1 \%$ of baseline, $\mathrm{n}=7$ slices, $p<$ 0.05 vs. control; For $0.3 \mu \mathrm{M}$ Ro25-6981, $116.1 \pm 13.4 \%$ of baseline, $\mathrm{n}=8$ slices, $p<0.05$ vs. control).

Bath application of the NR2A relatively selective antagonist NVP-AAM077 $(0.3 \mu \mathrm{M})$ also reduced LTP induced by spike-timing protocol (Figure 1F: $108.9 \pm 12.1 \%$ of baseline, $\mathrm{n}=7$ slices, $p<0.05$ vs. control). These results provide evidence that both NR2B- and NR2A-NMDARs contribute to LTP induced by spike-timing protocol.

\section{LTP induced by pairing protocol}

Next, we examined the effect of NR2B inhibition on LTP induced by pairing protocol (Figure 2A). Whole-cell recordings were done in CA1 pyramidal cells. The pairing protocol induced a significant potentiation of synaptic responses (Figure 2B: $192.1 \pm 22.0 \%$ of baseline, $\mathrm{n}=6$ slices; $p<0.05 v$ s. baseline). Bath application of $0.5 \mu \mathrm{M}$ Ro25-6981 did not affect the synaptic potentiation (Figure 2C: $181.6 \pm 22.8 \%$ of baseline, $\mathrm{n}=5$ slices; $p<0.05 v$ s. baseline). As shown in Figure 2D, there was no significant difference in LTP amplitudes in the presence and absence of Ro25-6981 (25-30 min post-induction, $p>0.05$ for Ro25-6981 vs. control). This result indicates that NR2BNMDARs are not required for LTP induced by the pairing protocol in the CA1 region, consistent with the previous report by Liu et al. [9]

\section{LTP induced by high-frequency stimulation}

We then investigated the effect of NR2B-NMDAR blockade on LTP induced by HFS using in vitro field-potential recordings. A two-train HFS induced a robust and sustained potentiation of synaptic responses in the CA1 region in control experiments (Figure 3B: $174.2 \pm 22.5 \%$ of baseline at 40-45 min post-HFS, $p<0.05 v s$. baseline, $\mathrm{n}=10$ slices). A similar amount of potentiation was observed in the presence of $0.5 \mu \mathrm{M}$ Ro25-6981 (Figure 3B: $172.3 \pm 15.5 \%$ of baseline, $p<0.05 v$ s. baseline; $p>0.05$ vs. control; $\mathrm{n}=7$ slices), indicating that LTP induced by HFS does not involve NR2B-NMDARs.

To address the possible role of NR2B-NMDARs in L-LTP, we used a four-train HFS to induce L-LTP in the CA1 region (Figure 4A). As shown in Figure 5B, the potentiation of filed EPSP was robust and sustained for over $3 \mathrm{~h}$ after the delivery of the HFS $(209.6 \pm 57.0 \%$ of baseline at 170-180 min post-HFS $p<0.05 v$ s. baseline, $\mathrm{n}=7$ slices). Bath application of $0.5 \mu \mathrm{M}$ Ro25-6981 did not affect the L-LTP (Figure 4B: $200.7 \pm 33.5 \%$ of baseline; $p<0.05 v$ s. baseline; $p>0.05 v s$. control, $\mathrm{n}=5$ slices). This result indicates that NR2B-NMDARs are not required for the L-LTP.

\section{$\mathrm{Ca}^{2+}$ signals triggered by spike-timing and pairing protocols} Calcium influx into postsynaptic neurons is a critical event in synaptic plasticity in the CA1 region. Here, we examined the intracellular $\mathrm{Ca}^{2+}$ signals elicited by spiketiming and pairing protocols and evaluated the contributions of NR2B-NMDARs using calcium imaging. The two protocols triggered an increase in $\left[\mathrm{Ca}^{2+}\right]_{\mathrm{i}}$ with different kinetics (Figure 5C and 5D, upper). Treatment with ifenprodil $(3 \mu \mathrm{M})$ reduced the $\mathrm{Ca}^{2+}$ signals under both protocols (Figure 5C and 5D, middle). By calculating the difference of the signals in control and in the presence of ifenprodil, we obtained the NR2B-NMDAR mediated $\mathrm{Ca}^{2+}$ signals (Figure 5C and 5D, lower). As shown, the NR2BNMDAR mediated calcium signal under the spike-timing protocol was much different in kinetics but not in absolute quantity from that under the pairing protocol. This difference in kinetics of $\mathrm{Ca}^{2+}$ efflux might help explain why NR2B-NMDARs are required for LTP induced by spike-timing protocol but not pairing protocol. It may be possible that the fluctuation of the NR2B-mediated $\mathrm{Ca}^{2+}$ efflux is a critical factor for induction of LTP under the spike-timing protocol.

\section{Acquisition of contextual fear memory}

A previous study in our laboratory showed that intra-CA1 blockade of NR2B-NMDARs with Ro25-6981 $(5.0 \mu \mathrm{g})$ had no effect on the acquisition of contextual fear memory in both rats and mice [24]. In that study, we trained the animals with a single CS-US pairing. Here, we reexamined the effect of intra-CA1 blockade of NR2BNMDARs on the acquisition of contextual fear memory using another selective NR2B-NMDAR antagonist ifenprodil. We also investigated the effect of intra-CA1 blockade of NR2A-NMDARs, using the relatively selective NR2A-NMDAR antagonist NVP-AAM077.

Intra-CA1 infusion of NVP-AAM077 $(0.012 \mu \mathrm{g}$ in $1 \mu \mathrm{l}$ PBS, $\mathrm{n}=6$ rats; $0.12 \mu \mathrm{g}$ in $1 \mu \mathrm{l} \mathrm{PBS,} \mathrm{n}=8$ rats), ifenprodil $(0.2 \mu \mathrm{g}$ in $1 \mu \mathrm{l}$ PBS, $\mathrm{n}=7 \mathrm{rats})$, or Ro25-6981 (5.0 $\mu \mathrm{g}$ in 1 $\mu \mathrm{l}$ PBS, $\mathrm{n}=8$ rats) was performed $15 \mathrm{~min}$ before the animals received the single CS-US pairing protocol. PBS was 


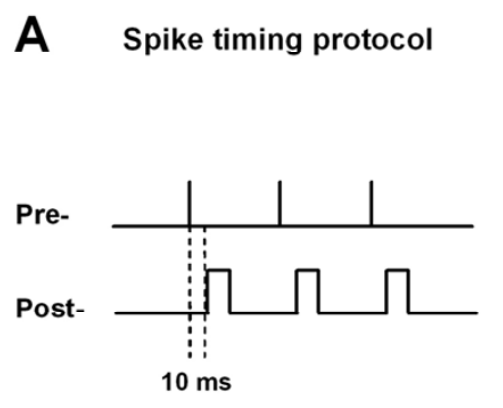

3 EPSPs with 3 APs at $30 \mathrm{~Hz}$

C Ifenprodil $(3 \mu \mathrm{M})$

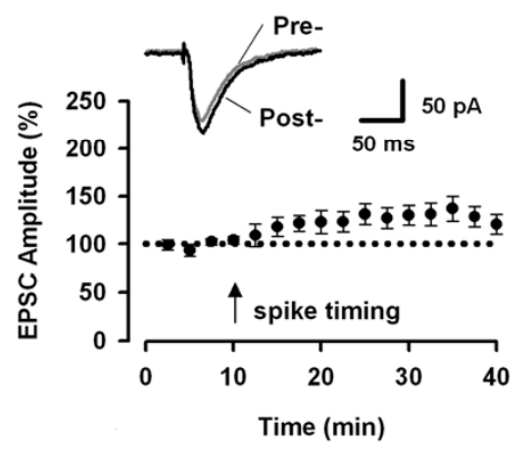

E

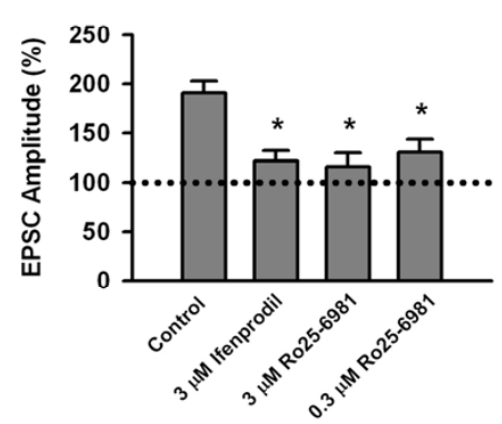

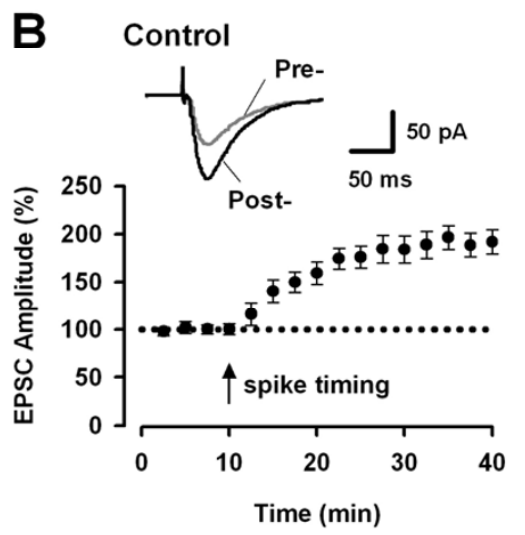

$\begin{array}{lll}- & \operatorname{Ro25-6981}(3 \mu \mathrm{M}) \\ & \circ & \operatorname{Ro25}-6981(0.3 \mu \mathrm{M})\end{array}$

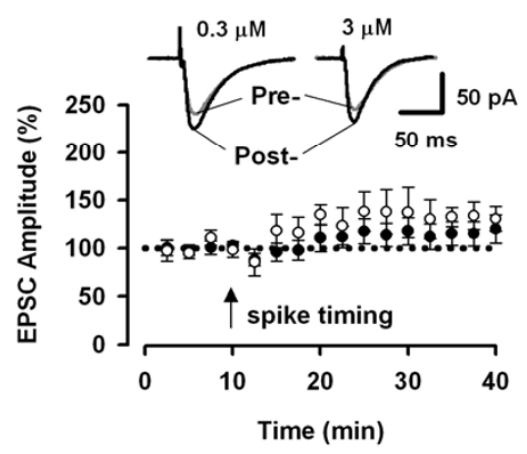

F $\quad$ NVP-AAM077 $(0.3 \mu \mathrm{M})$

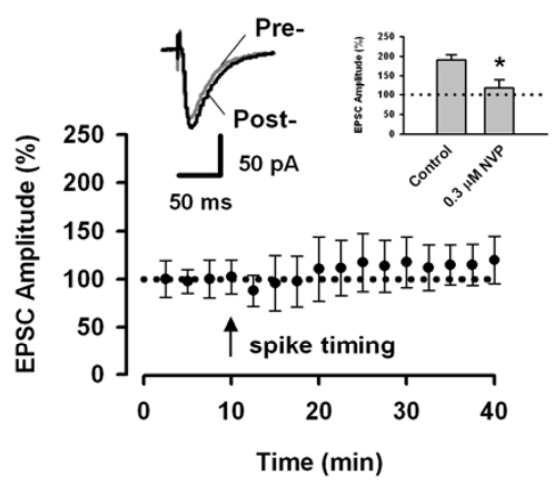

\section{Figure I}

NR2B-NMDARs are required for LTP induced by spike-timing protocol in area CAI. A. Schematic diagram of the spike-timing protocol. B. Spike-timing protocol, as indicated by the arrow, induced a significant LTP in CAI pyramidal neurons $(n=8)$. Sample traces of EPSC are the averages of 7 consecutive responses recorded during 5-10 min and 25-30 min, respectively. C. Bath application of ifenprodil partially blocked the LTP. $n=8$ neurons D. Bath application of Ro25-698I partially blocked the LTP. $\mathrm{n}=7$ neurons for $0.3 \mu \mathrm{M}$ Ro25-698I; $\mathrm{n}=8$ neurons for $3 \mu \mathrm{M}$ Ro25-698I. E. Histograms showing the effects of ifenprodil and Ro25-698I on the LTP. *p $<0.05$ vs. control. F. NR2A-NMDARs are required for LTP induced by spike-timing protocol in area CAI. Bath application of NVP-AAM077 blocked the LTP. $n=7$ neurons. inset: Histogram showing the effect of NVP-AAM077 on the LTP. * $p<0.05$ vs. control. 


\section{A Pairing protocol}

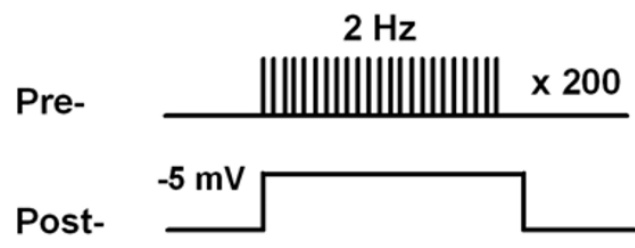

200 pulses at $2 \mathrm{~Hz}, \mathrm{~V}_{\mathrm{H}}=-5 \mathrm{mV}$

C $\quad \operatorname{Ro} 25-6981(0.5 \mu \mathrm{M})$

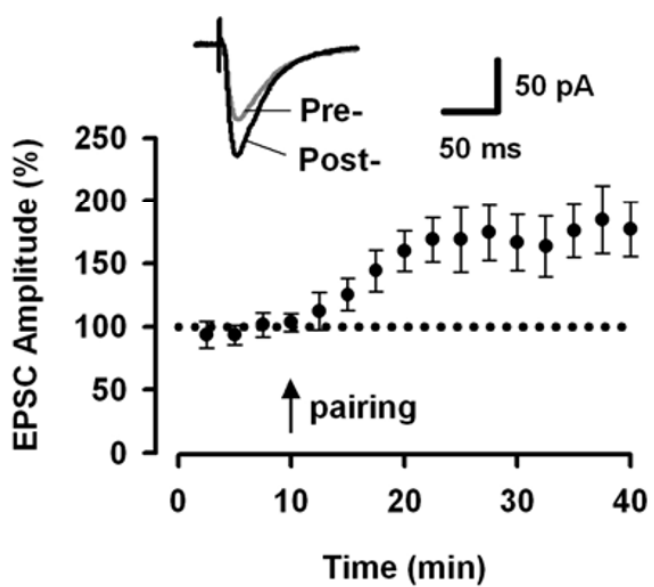

B Control

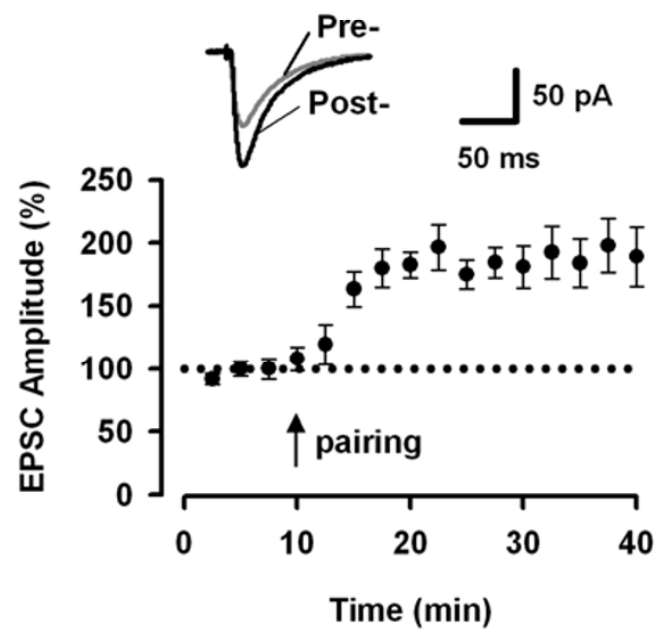

D

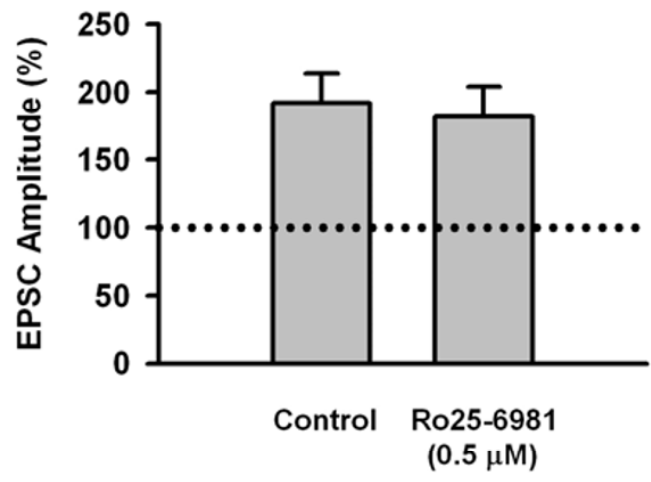

Figure 2

NR2B-NMDARs are not required for LTP induced by pairing protocol in area CAI. A. Schematic diagram of the pairing protocol. B. Pairing protocol, as indicated by the arrow, induced a significant LTP in CAI pyramidal neurons $(n=6)$. Sample traces of EPSC are the averages of 7 consecutive responses recorded during 5-10 min and 25-30 min, respectively. C. Bath application of Ro25-698I ( $0.5 \mu \mathrm{M})$ had no effect on the LTP $(n=5)$. D. Histogram showing the effect of Ro25-698I on the LTP. p $>0.05$ vs. control. 


\section{A HFS protocol (LTP)}
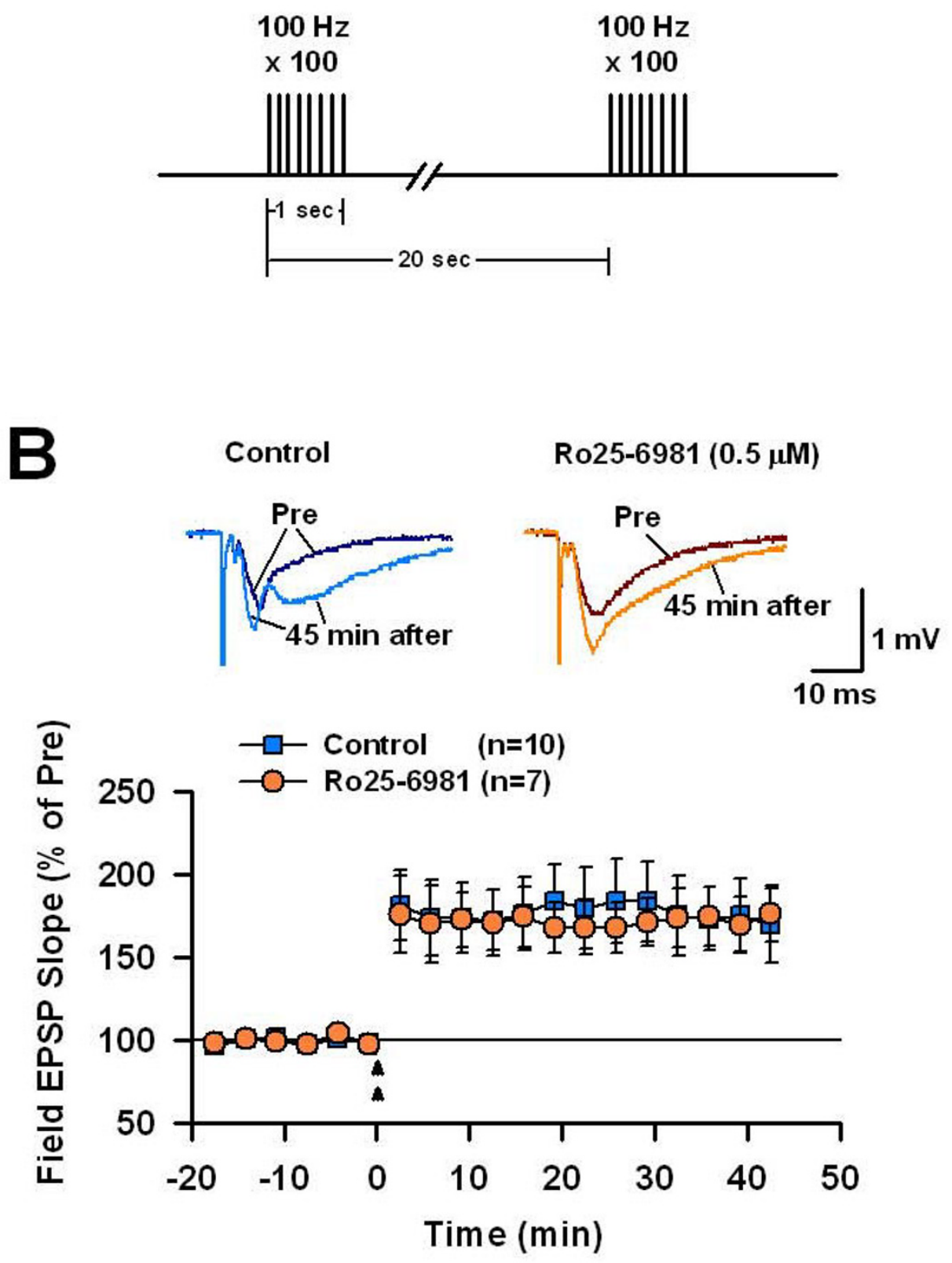

Figure 3

NR2B-NMDARs are not required for LTP induced by HFS protocol in area CAI. A. Schematic diagram of the high frequency stimulation (HFS; 2 train). B. LTP of field EPSP induced by the HFS in control ( $n=10$ slices) and in the presence of Ro25-698I ( $n=7$ slices). Ro25-798I had no effect on the LTP. 


\section{A HFS protocol (L-LTP)}

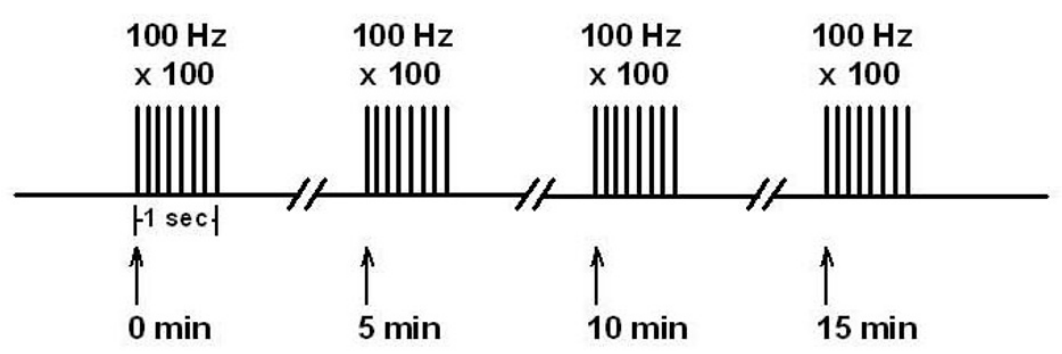

B

Control

$\operatorname{Ro} 25-6981(0.5 \mu \mathrm{M})$
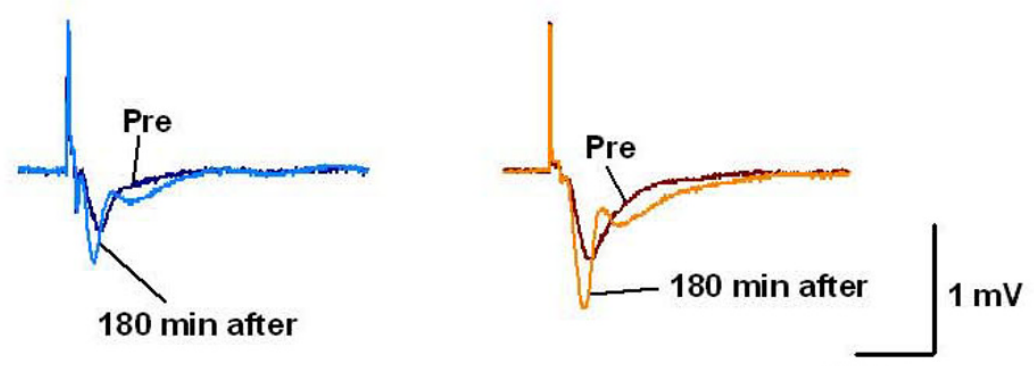

$10 \mathrm{~ms}$

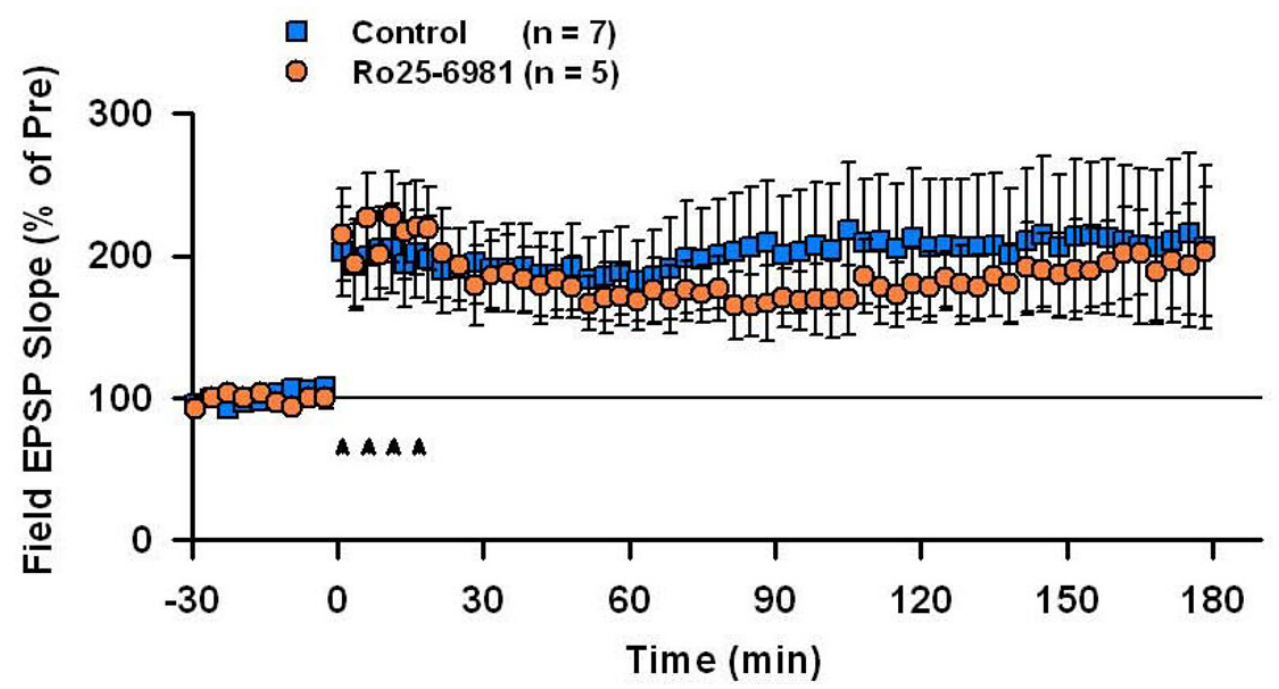

Figure 4

NR2B-NMDARs are not required for L-LTP induced by HFS protocol in area CAI. A. Schematic diagram of the high frequency stimulation (HFS; 4 trains). B. Late phase LTP of field EPSP induced by the HFS in control ( $n=7$ slices) and in the presence of Ro25-698I ( $n=5$ slices). Ro25-798I had no effect on the L-LTP. 
A

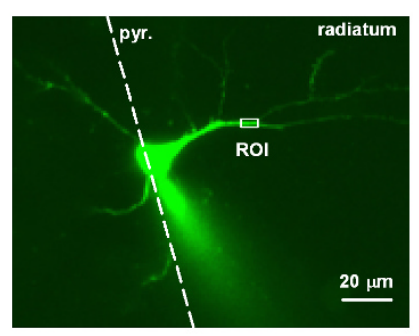

C
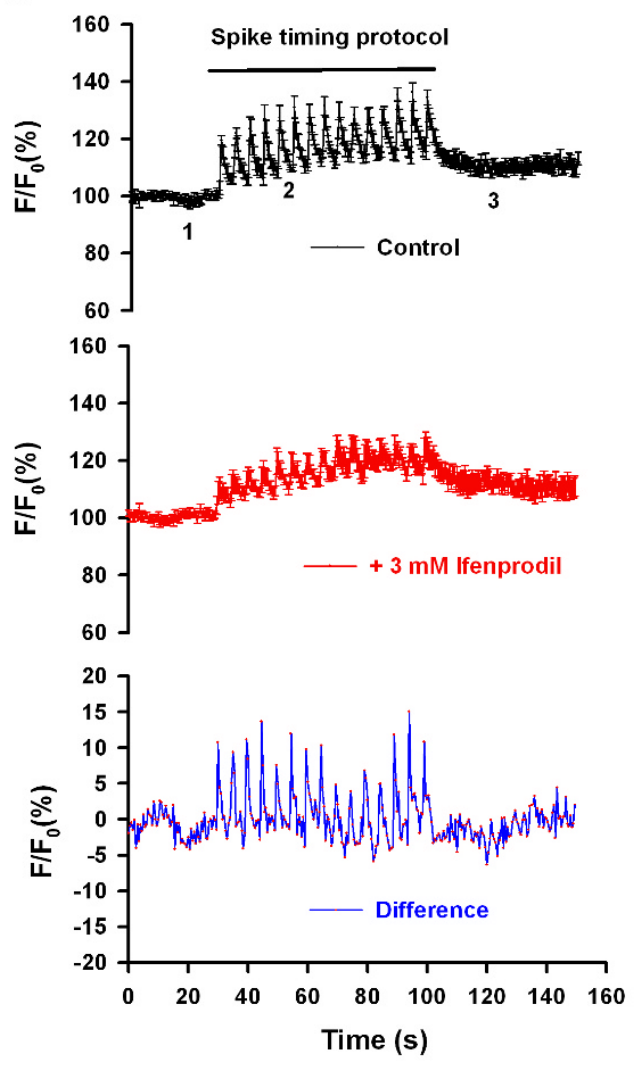

B

1

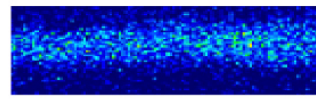

2

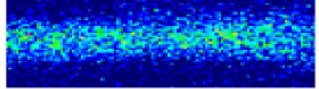

3

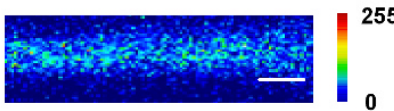

D
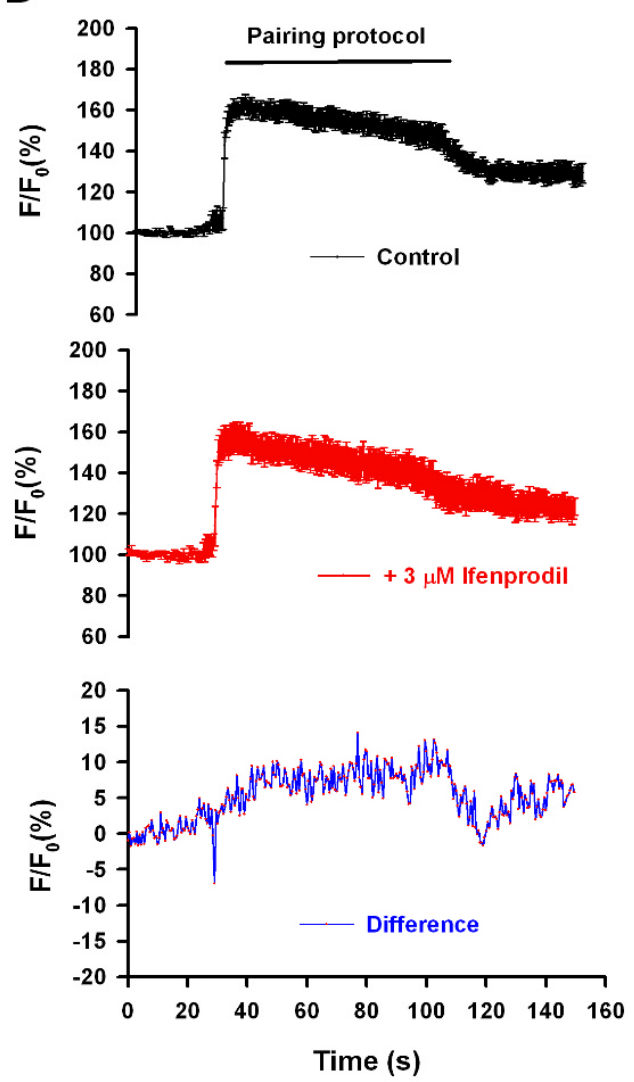

\section{Figure 5}

NR2B-NMDAR mediated $\mathrm{Ca}^{2+}$ influx under spiking-timing and pairing protocols. A. A representative image showing the CAI pyramidal neuron filled with OGB-I. ROI, region of interests. B. Raw sample fluorescence images from the ROI before (I), during (2) and after (3) induction of LTP with the spike-timing protocol. Scale bar, I.0 $\mu \mathrm{m}$. C. Elevation of Ca2+ signal in the ROI during induction of LTP with the spike-timing protocol (upper). Treatment with ifenprodil ( $3 \mu \mathrm{M})$ reduced the $\mathrm{Ca} 2+$ signal (middle). The difference of $\left[\mathrm{Ca}_{2}+\right]$ signals in control and in the presence of ifenprodil (lower) shows the NR2BNMDAR mediated $\mathrm{Ca} 2+$ influx. D. Elevation of $\mathrm{Ca} 2+$ signal in the ROI during induction of LTP with the pairing protocol (upper). Treatment with ifenprodil $(3 \mu \mathrm{M})$ reduced the $\mathrm{Ca} 2+$ signal (middle). The difference of $\left[\mathrm{Ca}_{2}+\right]$ signals in control and in the presence of ifenprodil (lower) shows the NR2B-NMDAR mediated Ca2+ influx. 
similarly infused as vehicle control ( $1 \mu \mathrm{l} ; \mathrm{n}=9$ rats $)$. Fear memory was tested $48 \mathrm{~h}$ post-conditioning. As shown in Figure 6A1, rats with intra-CA1 infusion of ifenprodil demonstrated no difference in contextual freezing score in relative to vehicle controls $(\mathrm{F}(1,14)=0.23, p>0.05$ for ifenprodil vs. vehicle). By contrary, rats with intra-CA1 infusion of NVP-AAM077 exhibited a severe deficit in contextual fear memory $(\mathrm{F}(1,13)=7.93, p<0.01$ for $0.012 \mu \mathrm{g}$ NVP-AAM077 vs. vehicle; $\mathrm{F}(1,15)=6.13, p<$ 0.05 for $0.12 \mu \mathrm{g}$ NVP-AAM077 vs. vehicle). Auditory fear memory was tested $1 \mathrm{~h}$ after contextual fear memory was tested. As shown in Figure 6A2, rats with intra-CA1 blockade of NR2B- and NR2A-NMDARs showed no deficit in auditory fear memory $(\mathrm{F}(4,31)=0.60, p>0.05)$. Thus, the acquisition of contextual fear memory induced by the single CS-US pairing involves NR2A- but not NR2BNMDARs in the CA1 region.

Considering that the importance of NR2B-NMDARs for LTP induction is protocol-dependent, we attempted to know whether the role of NR2B-NMDARs in memory acquisition is also dependent on training paradigms. To address this, we examined the effects of intra-CA1 inhibition of NR2B- as well as NR2A-NMDARs on the acquisition of contextual fear memory induced by the five CS-US pairing conditioning. Our pilot experiment showed that the five CS-US pairing protocol could induce more freezing responses than the single CS-US pairing protocol did (Table 1).

Intra-CA1 injection of NVP-AAM077 $(0.012 \mu \mathrm{g}$ in $1 \mu \mathrm{l}$ PBS, $\mathrm{n}=6$ rats), ifenprodil $(0.2 \mu \mathrm{g}, \mathrm{n}=7$ rats $)$, or Ro256981 ( $5.0 \mu \mathrm{g}, \mathrm{n}=8$ rats) was performed $15 \mathrm{~min}$ before the animals was trained with the five CS-US pairing protocol. PBS was similarly infused as vehicle control $(1 \mu \mathrm{l}, \mathrm{n}=7$ rats). Fear memory was tested $48 \mathrm{~h}$ post-conditioning. As shown in Figure 6B1, rats treated with NVP-AAM077, ifenprodil or Ro25-6981 showed a deficient contextual fear memory as compared with vehicle controls $(\mathrm{F}(1,11)=$ $35.3, p<0.01$ for NVP-AAM077 vs. vehicle; $F(1,12)=$ $31.21, p<0.01$ for ifenprodil vs. vehicle; $\mathrm{F}(1,13)=26.05$, $p<0.01$ for Ro25-6981 vs. vehicle). However, the rats with intra-CA1 blockade of NR2B- and NR2A-NMDARs showed a normal auditory fear memory (Figure 6B2: $\mathrm{F}(3,24)=0.72, p>0.05)$. Thus, both NR2A- and NR2BNMDARs in the CA1 region are required for the acquisition of contextual fear memory induced by the five CS-US pairing conditioning.

\section{Retrieval of contextual fear memory}

As just described, NR2B-NMDARs in the CA1 region are involved in the acquisition of contextual fear memory in a conditioning-strength dependent way. Here, we wanted to know if NR2B-NMDARs are involved in the retrieval of contextual fear memory in a similar way.
First, we examined the effects of intra-CA1 blockade of NR2B- as well as NR2A-NMDARs on memory retrieval for the single CS-US pair conditioning. NVP-AAM077 (0.012 $\mu \mathrm{g}$ in $1 \mu \mathrm{l} \mathrm{PBS}, \mathrm{n}=6$ rats; $0.12 \mu \mathrm{g}$ in $1 \mu \mathrm{l} \mathrm{PBS}, \mathrm{n}=7$ rats), ifenprodil $(0.2 \mu \mathrm{g}$ in $1 \mu \mathrm{l}$ PBS, $\mathrm{n}=8$ rats $)$, or Ro25-6981 (5.0 $\mu \mathrm{g}$ in $1 \mu \mathrm{l} \mathrm{PBS}, \mathrm{n}=6 \mathrm{rats})$ was infused into the CA1 region $15 \mathrm{~min}$ before memory retention was tested. PBS was similarly infused as vehicle control $(1 \mu \mathrm{l}, \mathrm{n}=9$ rats). As shown in Figure 7A1, An one-way ANOVA revealed a significant group effect on contextual freezing scores $(\mathrm{F}(4,31)=8.12, p<0.05)$. Planned comparison showed that the rats treated with NVP-AAM077, ifenprodil, or Ro25-6981 exhibited a deficient contextual fear memory $(\mathrm{F}(1,13)=15.48, p<0.01$ for $0.012 \mu \mathrm{g}$ NVP-AAM077 vs. vehicle; $F(1,14)=10.61, p<0.01$ for $0.12 \mu \mathrm{g}$ NVPAAM077 vs. vehicle; $F(1,15)=20.71, p<0.01$ for ifenprodil vs. vehicle; $\mathrm{F}(1,13)=13.23, p<0.01$ for Ro256981 vs. vehicle). However, each group of rats demonstrated a normal auditory fear memory (Figure 7A2: F(4, 21) $=0.62, p>0.05)$.

Then, we examined the effects of intra-CA1 inhibition of NR2B- as well as NR2A-NMDARs on memory retrieval for the five CS-US pairing conditioning. NVP-AAM077 $(0.012 \mu \mathrm{g}$ in $1 \mu \mathrm{l}$ PBS, $\mathrm{n}=6$ rats $)$, ifenprodil $(0.2 \mu \mathrm{g}$ in 1 $\mu \mathrm{l}$ PBS, $\mathrm{n}=6$ rats), or Ro25-6981 (5.0 $\mu \mathrm{g}$ in $1 \mu \mathrm{l} \mathrm{PBS,} \mathrm{n}=$ 5 rats) was infused into the CA1 region 15 min before memory retention test. PBS was similarly injected as vehicle control ( $1 \mu \mathrm{l}, \mathrm{n}=7$ rats). As shown in Figure 7B1, an one-way ANOVA revealed a significant group effect on contextual freezing scores $(\mathrm{F}(3,20)=8.12, p<0.01)$. Planned comparison showed that the rats treated with NVP-AAM077, ifenprodil, or Ro25-6981 demonstrated a deficient contextual fear memory $(\mathrm{F}(1,11)=19.66$, $p<$ 0.01 for NVP-AAM077 vs. vehicle; $F(1,11)=12.10$, $p<$ 0.01 for ifenprodil vs. vehicle; $F(1,10)=13.06, p<0.01$ for Ro25-6981 vs. vehicle). Auditory fear memory was intact upon the drug infusion (Figure 7B2: $\mathrm{F}(3,16)=0.22$, $p>0.05)$.

Taken together, these results strongly suggest that NR2BNMDARs, as well as NR2A-NMDARs, in the CA1 region are required for the retrieval of contextual fear memory induced by the one and five CS-US pairing protocols.

\section{Discussion}

The present study shows that NR2B-NMDARs are required for LTP induced by the spike-timing protocol, but not for LTP induced by the pairing or HFS protocol. Intracellular $\mathrm{Ca}^{2+}$ signals triggered by spiking-timing and pairing protocols display different kinetics. Late-phase LTP induced by HFS protocol does not involve NR2B-NMDARs. Moreover, the significance of NR2B-NMDARs in acquisition of contextual fear memory is dependent on conditioning protocols. 


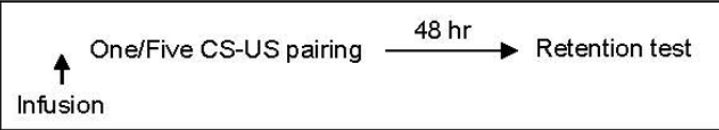

\section{A1}

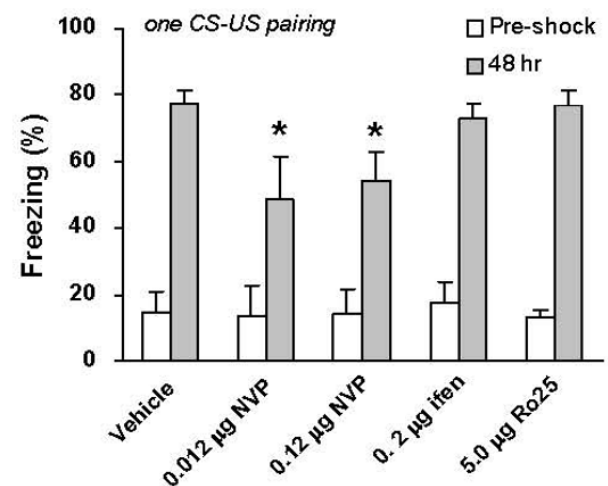

A2

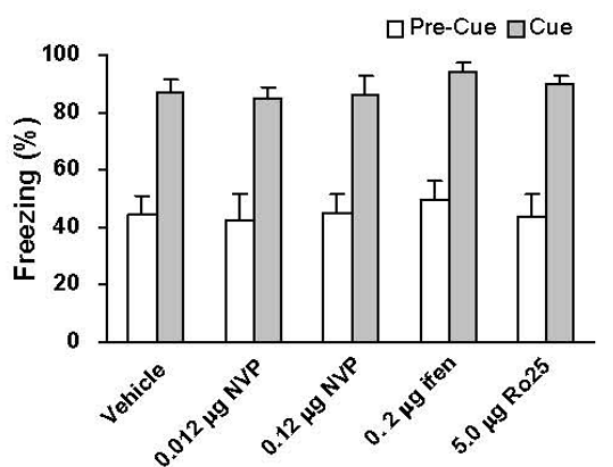

C

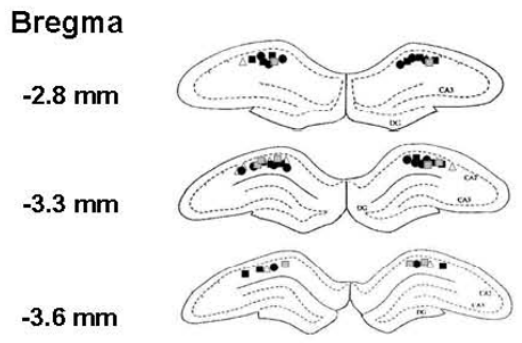

B1 Contextual Memory

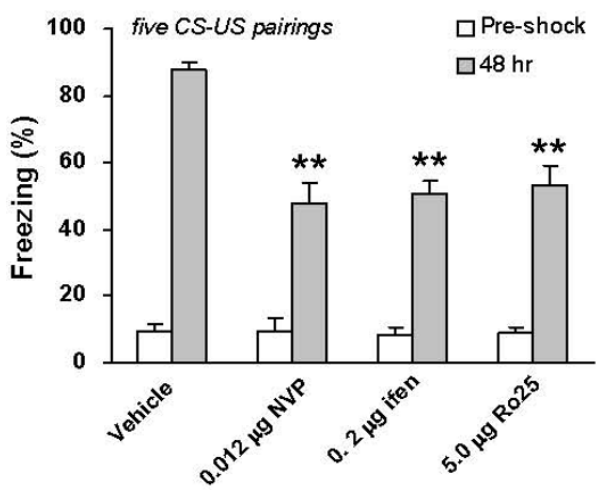

B2 Auditory Memory

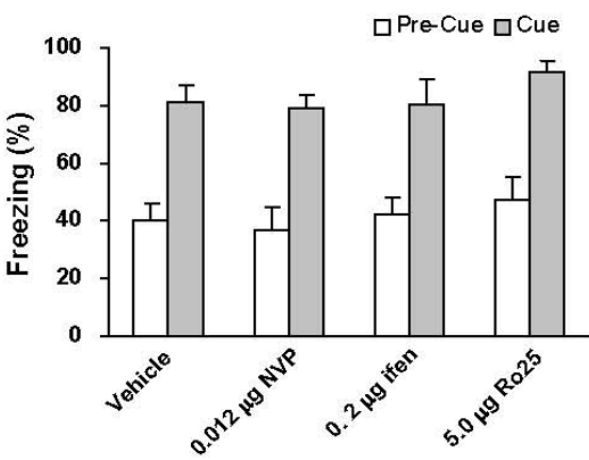

D

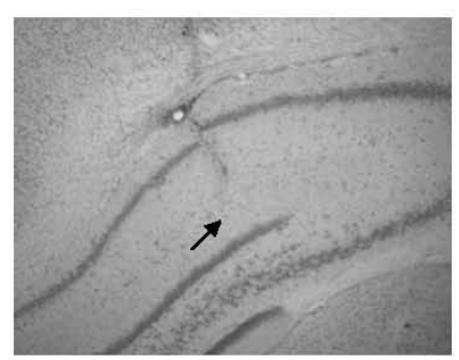

\section{Figure 6}

NR2B-NMDARs are required for the acquisition of contextual fear memory induced by the five but not one CS-US pairing conditioning. A. Pre-training intra-CAI inhibition of NR2B-NMDARs had no impact on, while inhibition of NR2A-NMDARs impaired 48-h contextual fear memory induced by the single CS-US pairing protocol (AI). The acquisition of auditory fear memory was intact (A2). The result with Ro25-698I was reported in the previous study (Zhao et al., 2005). *p < 0.05 vs. vehicle B. Pre-training intra-CAI inhibition of NR2B- or NR2A-NMDARs impaired 48-h contextual fear memory induced by the five CS-US pairing protocol $(\mathrm{BI})$. The acquisition of auditory fear memory was intact (B2). ** $p<0.0 \mathrm{I}$ vs. vehicle. C. Reconstruction of the infusion sites in the CAI region. Filled squares: vehicle; Open circles: $0.012 \mu \mathrm{g}$ NVP-AAM077; Filled circles: $0.12 \mu \mathrm{g}$ NVP-AAM077; Open triangles: ifenprodil; Grey squares: Ro25-698I. D. A representative coronal section showing an infusion site of ifenprodil in the CAI region. 
Table I: Contextual fear memory induced by the one and five CS-US pairing protocols

\begin{tabular}{ccc}
\hline Protocol & Pre-shock & 48-h Retention Test \\
\hline Protocol I: I CS-US (US: I.0 mA, 2.0 s) & $11.27 \pm 4.65$ & $72.86 \pm 3.20$ \\
Protocol 2: 5 CS-US (US: I.0 mA, 0.5 s) & $9.53 \pm 2.05$ & $90.00 \pm 1.77 * *$ \\
\hline
\end{tabular}

Data is expressed as the percentage of freezing (mean \pm SEM). Protocol-I: $n=7$; Protocol-2: $n=6 . * * p<0.01$ vs. Protocol I, $t$-test.

\section{Role of NR2B-NMDARs in LTP}

Many previous studies using different experimental approaches have shown that NR2B-NMDARs contribute to synaptic potentiation in different areas of the central nervous system [12-14,24-26]. However, some other studies have reported that NR2B-NMDARs are not required for synaptic potentiation or LTP $[9,10]$. Considering that NR2B- and NR2A- NMDARs have different biophysical properties and couple to different intracellular signaling cascades [8,26-29], it may be possible that different induction protocols may activate different NMDAR subtypes. Indeed, it has been reported that different LTP-inducing protocols recruit different signaling pathways. For example, in the amygdala, pairing-protocol induced LTP depends on L-type voltage-gated calcium channels (LVGCCs) but not NMDARs, while tetanus-stimulation induced LTP involves NMDARs but not L-VGCCs [30]. In the present study, we demonstrate that the involvement of NR2B-NMDARs in LTP is dependent on induction protocols. NR2B-NMDARs are required for LTP induced by the spike-timing protocol, but not by the pairing protocol. Although it has been reported that a low dose Ro25-6981 $(0.3 \mu \mathrm{M})$ enhances, but not reduces NMDAR-mediated EPSCs in the CA1 region [24,31], the same dose Ro256981 in the present study reduced LTP induced by the spike timing protocol, but not by the pairing protocol (Table 2).

The induction of spike timing-dependent LTP requires activation of NMDAR $[20,21]$. Spike timing-dependent potentiation requires the temporal window between presynaptic spikes and postsynaptic EPSPs. It is known that NR2B- and NR2A-NMDARs have different characteristics: NR2B-NMDARs have slower kinetics than NR2ANMDARs [27]. It is possible that NR2B-NMDARs are more sensitive to the certain form of LTP induction protocol. Berberich et al. reported that the amount of charge transfer during LTP induction is a critical factor in low-frequency stimulation pairing [31]. Thus, there is such a possibility, although remains to be demosntrated, that inhibition of NR2B-NMDARs affect the charge transfer during LTP induction.

The induction-protocol dependent involvement of NR2BNMDARs in LTP suggests that LTP induced by different protocols may have different intracellular mechanisms. Indeed, it has been suggested that different LTP induction protocols may activate distinct signaling cascades that generate LTP with different expression mechanisms [3234]. LTP induction in the CA1 region requires $\mathrm{Ca}^{2+}$ influx through NMDAR, either through direct activation of $\mathrm{Ca}^{2+}$ sensitive substrates and/or subsequent $\mathrm{Ca}^{2+}$ release from intracellular $\mathrm{Ca}^{2+}$ stores $[1,35]$. $\mathrm{Ca}^{2+}$ influx activates calcium/calmodulin-dependent protein kinase II (CaMKII), which is required as a critical cellular cascades for NMDAdependent LTP $[11,36]$. Recently, Gerkin et al. reported that NMDAR subtypes can differentially activate competitive signaling modules in the induction and integration of spike-timing-dependent plasticity [37]. That is, $\mathrm{Ca}^{2+}$ influx via NR2B-NMDARs may activate either CaMKII or calcineurin $(\mathrm{CaN})$, which is dependent on the outcome of dynamic competition driven by NMDA receptor subtypes. Our data suggested that $\mathrm{Ca}^{2+}$ influx via NR2B-NMDARs induced by spike-timing protocol can activate CaMKII and lead to LTP. Furthermore, our calcium imaging in the present study showed that the NR2B-NMDAR mediated $\mathrm{Ca}^{2+}$ transients were faster under the spike-timing than pairing protocols, which might explain the different significance of NR2B-NMDARs in LTP under the two protocols since fast $\mathrm{Ca}^{2+}$ transients are better for LTP, but slow $\mathrm{Ca}^{2+}$ transients not [38].

\section{Role of NR2B-NMDARs in memory}

It has been documented that NR2B-NMDARs in the hippocampus, amygdala and anterior cingulate cortex play an important role in memory or persistent pain $[14,24,39,40]$. Genetic over-expression of NR2B receptor subtype in the forebrain enhances spatial memory as well as contextual fear memory [14], whereas pharmacological blockade of NMDARs in the CA1 region impairs contextual fear memory [19]. Pharmacological blockade of NR2B-NMDARs in the lateral amygdala or in the ACC impairs formation of fear memory $[23,24]$. However, it is unclear whether hippocampal NR2A- and NR2BNMDARs have differential roles in fear memory formation.

The present study shows that, unlike NR2A-NMDARs that are required non-differentially, NR2B-NMDARs are involved in acquisition of contextual fear memory in a conditioning-strength dependent way. Pre-conditioning intra-CA1 infusion of the NR2B selective antagonist ifenprodil or Ro25-6981 impaired contextual fear memory induced by five but not one CS-US pairing protocol, while 


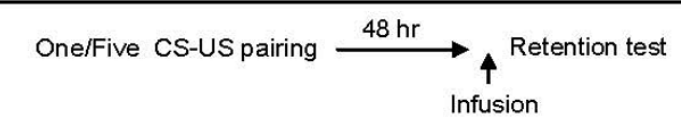

\section{A1}

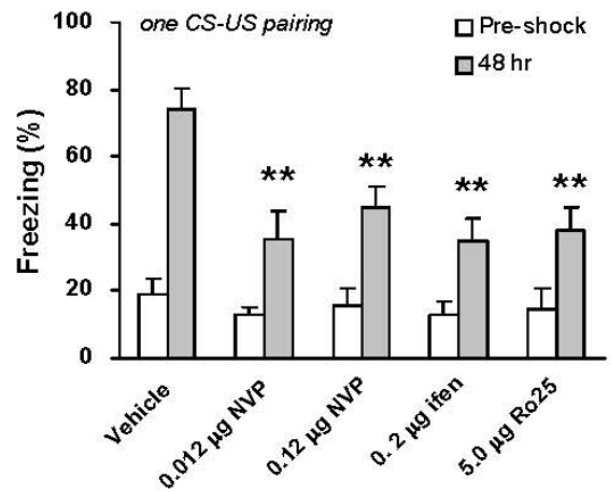

A2

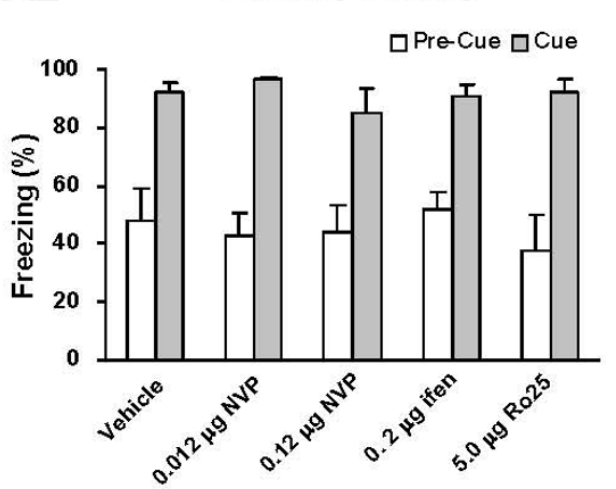

C

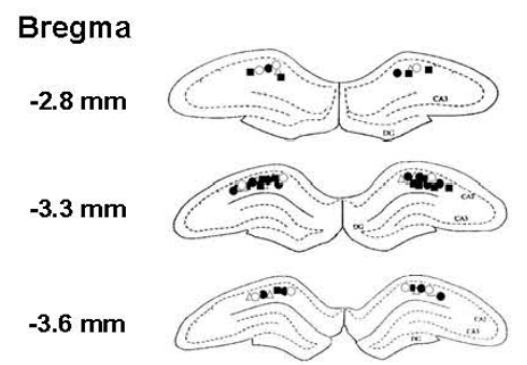

B1 Contextual Memory

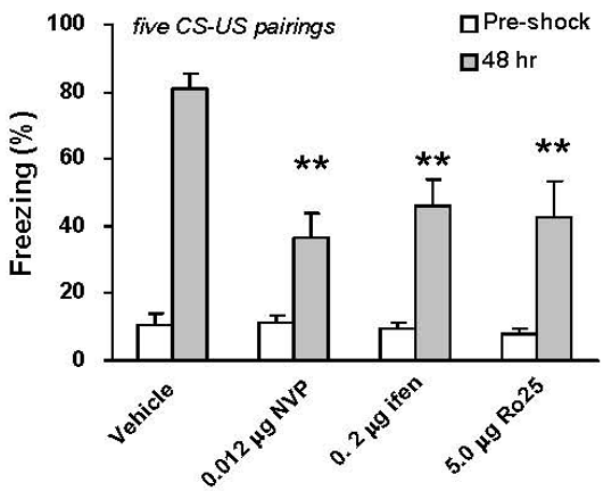

B2

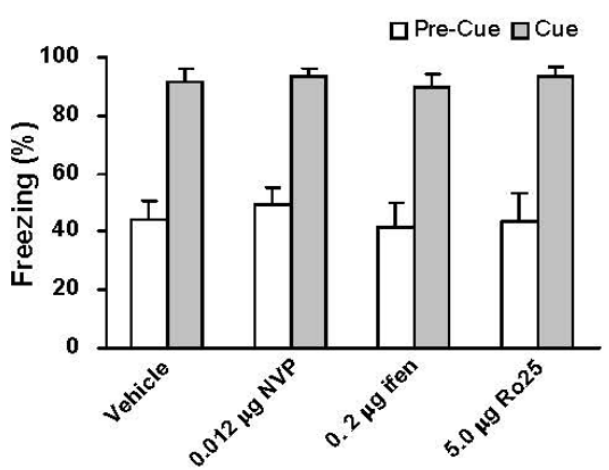

D

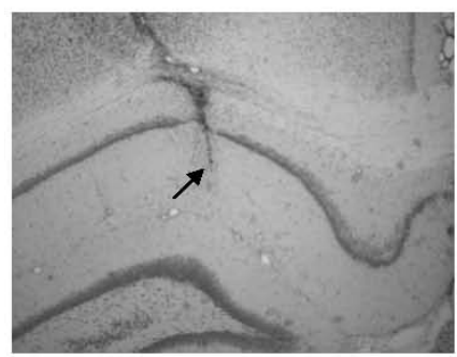

\section{Figure 7}

NR2B-NMDARs are required for the retrieval of contextual fear memory induced by both five and one CS-US pairing conditioning. A. Pre-retrieval intra-CAI inhibition of NR2B- or NR2A-NMDARs impaired the expression of 48-h contextual fear memory induced by the single CS-US pairing protocol (AI). The expression of 48-h auditory fear memory was intact (A2). **p $<0.0$ l vs. vehicle. B. Pre-retrieval intra-CAI inhibition of NR2B- or NR2A-NMDARs impaired the expression of 48-h contextual fear memory induced by the five CS-US pairing protocol (BI). The expression of 48-h auditory fear memory was intact (B2). $* * p<0.01$ vs. vehicle. C. Reconstruction of the infusion sites in the CAI region. Filled squares: vehicle; Filled circles: NVP-AAM077; Open triangles: ifenprodil; Open circles: Ro25-698I. D. A representative coronal section showing an infusion site of ifenprodil in the CAI region. 
Table 2: Effects of Ro25-698 I on LTP induced by different protocols in the anterior cingulate cortex (ACC) and hippocampal CA I region

\begin{tabular}{lcc}
\hline Induction Protocol & ACC (Zhao et al., 2005) & CAl (the present study) \\
\hline Spike timing protocol & LTP blocked & LTP reduced or blocked \\
Pairing protocol & LTP reduced & Normal LTP \\
HFS protocol & -- & Normal LTP \\
\hline
\end{tabular}

similar treatment with the NR2A antagonist NVPAAM077 disrupted memory for both protocols. This is well consistent with a recent study in our laboratory [41], showing that NR2B-NMDARs in the basolateral nucleus of amygdala (BLA) are involved in acquisition of auditory fear memory also in a conditioning-strength dependent way. In that study, we reported that pre-conditioning intra-BLA infusion of the NR2B selective antagonist ifenprodil or Ro25-6981 impaired auditory fear memory induced by five but not one CS-US pairing protocol, while similar treatment with the NR2A antagonist NVPAAM077 interfered with memory for both protocols. Consistently, genetic over-expression of NR2B C-terminal in the BLA, which interferes with the C-terminal mediated intracellular signaling, produced a severe deficit in auditory fear memory for five but not one CS-US pairing protocol, whereas over-expression of NR2A C-terminal produced a deficient memory for both protocols. There seems a recruitment mechanism in the hippocampus and amygdala for involvement of NR2B-NMDARs in memory acquisition: the heavier the training load is, the more involved the NR2B-NMDAR is.

\section{Selectivity of NVP-AAM077}

NR2A- and NR2B-NMDARs have distinct electrophysiological and signaling properties [8,27,42-48]. Dissection of the functions of NR2A- and NR2B-NMDARs will promote our understanding of their roles in learning and memory. As specific antagonists for NR2A-NMDAR are not available, the role of NR2A-NMDARs in learning and memory is still poorly understood. Recently, a relatively selective NR2A-NMDAR antagonist, NVP-AAM077, has been developed. However, some previous studies argue that NVP-AAM077 is not sufficient to discriminate between NR2A- and NR2B-NMDARs, with about 10-fold higher selectivity for NR2A- than for NR2B-NMDARs $[49,50]$.

In the present study, we used NVP-AAM077 (0.012 or $0.12 \mu \mathrm{g}$ in $1.0 \mu \mathrm{l}$ vehicle) for pharmacological blockade of NR2A-NMDARs in the CA1 region. We found that treatment with NVP-AAM077 non-differentially impaired the acquisition of fear memory induced by the two conditioning protocols, one of which included a single CS-US pairing and the other five CS-US pairings. Although NVPAAM077 could act at NR2B as well, it is unlikely that the
NVP-AAM077 effect was mediated by NR2B-NMDARs, because similar treatment with the selective NR2BNMDAR antagonist ifenprodil $(0.2 \mu \mathrm{g}$ in $1.0 \mu \mathrm{l}$ vehicle, equal molarity with $0.12 \mu \mathrm{g} / 1.0 \mu \mathrm{l}$ NVP-AAM077) or Ro25-6981 (5.0 $\mu \mathrm{g})$ impaired the acquisition of fear memory induced by the single CS-US pairing protocol. This result strongly suggests an essential role of NR2ANMDARs in the CA1 region in acquisition of contextual fear memory, regardless of conditioning strength.

In summary, the present study strongly suggests an induction- and conditioning-protocol dependent involvement of NR2B-NMDARs in the CA1 region in long-term potentiation and contextual fear memory formation.

\section{Competing interests}

The authors declare that they have no competing interests.

\section{Authors' contributions}

$\mathrm{X}-\mathrm{HZ}, \mathrm{L}-\mathrm{JW}, \mathrm{BG}$ and MR carried out the electrophysiological studies. $\mathrm{X}-\mathrm{HZ}$ also performed the behavior experiments. L-JW performed Ca imaging experiments. B-ML and $\mathrm{MZ}$ conceived of the study, and participated in its design and coordination. All authors read and approved the final manuscript.

\section{Acknowledgements}

This work was supported by grants to BML from the Ministry of Science and Technology of China (2006CB500807 and 2006AA02Z1 99), the Ministry of Education of China (Program for Changjiang Scholars and Innovative Research Team in University), and the National Natural Science Foundation of China (NSFC) (30225023 and 30430240), and to XHZ from the NSFC (307002 I8).

This work was also supported by grants to $M Z$ from the Canadian Institutes of Health Research, the EJLB-CIHR Michael Smith Chair in Neurosciences and Mental Health, the Canada Research Chair, and the NSFC (305280 I0), and the China-Canada Joint Health Research Initiative (NSFC grant 306 I I 20530 to BML and MZ).

\section{References}

I. Bliss TV, Collingridge GL: A synaptic model of memory: longterm potentiation in the hippocampus. Nature 1993, 36I(6407):31-39.

2. Malenka RC, Nicoll RA: NMDA-receptor-dependent synaptic plasticity: multiple forms and mechanisms. Trends Neurosci 1993, I6( I 2):52I-527.

3. Tsien JZ, Huerta PT, Tonegawa S: The essential role of hippocampal CAI NMDA receptor-dependent synaptic plasticity in spatial memory. Cell 1996, 87(7): I327-I338. 
4. Cull-Candy S, Brickley S, Farrant M: NMDA receptor subunits: diversity, development and disease. Curr Opin Neurobiol 200I, I I(3):327-335.

5. Dingledine R, Borges K, Bowie D, Traynelis SF: The glutamate receptor ion channels. Pharmacol Rev 1999, 5 I (I):7-6I

6. Kutsuwada T, Kashiwabuchi N, Mori H, Sakimura K, Kushiya E, Araki K, Meguro H, Masaki H, Kumanishi T, Arakawa M, Mishina M: Molecular diversity of the NMDA receptor channel. Nature 1992, 358(638I):36-4I.

7. Loftis JM, Janowsky A: The $\mathbf{N}$-methyl-D-aspartate receptor subunit NR2B: localization, functional properties, regulation, and clinical implications. Pharmacol Ther 2003, 97(I):55-85.

8. Monyer H, Sprengel R, Schoepfer R, Herb A, Higuchi M, Lomeli H, Burnashev N, Sakmann B, Seeburg PH: Heteromeric NMDA receptors: molecular and functional distinction of subtypes. Science 1992, 256(5060): $1217-1221$.

9. Liu L, Wong TP, Pozza MF, Lingenhoehl K, Wang Y, Sheng M, Auberson YP, Wang YT: Role of NMDA receptor subtypes in governing the direction of hippocampal synaptic plasticity. Science 2004, 304(5673): 1021-1024.

10. Massey PV, Johnson BE, Moult PR, Auberson YP, Brown MW, Molnar E, Collingridge GL, Bashir ZI: Differential roles of NR2A and NR2B-containing NMDA receptors in cortical long-term potentiation and long-term depression. J Neurosci 2004, 24(36):782I-7828.

II. Malenka RC, Nicoll RA: Long-term potentiation - a decade of progress? Science 1999, 285(5435): 1870-1874.

12. Weitlauf C, Honse Y, Auberson YP, Mishina M, Lovinger DM, Winder DG: Activation of NR2A-containing NMDA receptors is not obligatory for NMDA receptor-dependent long-term potentiation. J Neurosci 2005, 25(37):8386-8390.

13. Berberich S, Punnakkal P, Jensen V, Pawlak V, Seeburg PH, Hvalby O, Kohr G: Lack of NMDA receptor subtype selectivity for hippocampal long-term potentiation. I Neurosci 2005, 25(29):6907-6910.

14. Tang YP, Shimizu E, Dube GR, Rampon C, Kerchner GA, Zhuo M, Liu $G$, Tsien JZ: Genetic enhancement of learning and memory in mice. Nature 1999, 40 I(6748):63-69.

15. Ji J, Maren S: Differential roles for hippocampal areas CAI and CA3 in the contextual encoding and retrieval of extinguished fear. Learn Mem 2008, I5(4):244-25I.

16. Daumas S, Halley H, Frances B, Lassalle JM: Encoding, consolidation, and retrieval of contextual memory: differential involvement of dorsal CA3 and CAI hippocampal subregions. Learn Mem 2005, I 2(4):375-382.

17. Fanselow MS, Kim JJ, Yipp J, De Oca B: Differential effects of the $\mathrm{N}$-methyl-D-aspartate antagonist DL-2-amino-5-phosphonovalerate on acquisition of fear of auditory and contextual cues. Behav Neurosci 1994, 108(2):235-240.

18. Bast T, Zhang WN, Feldon J: Dorsal hippocampus and classical fear conditioning to tone and context in rats: effects of local NMDA-receptor blockade and stimulation. Hippocampus 2003, I3(6):657-675.

19. Melik E, Babar E, Ozen E, Ozgunen T: Hypofunction of the dorsal hippocampal NMDA receptors impairs retrieval of memory to partially presented foreground context in a single-trial fear conditioning in rats. Eur Neuropsychopharmacol 2006, 16(4):24I-247.

20. Bi GQ, Poo MM: Synaptic modifications in cultured hippocampal neurons: dependence on spike timing, synaptic strength, and postsynaptic cell type. J Neurosci 1998, 18(24): I0464-10472.

21. Markram H, Lubke J, Frotscher M, Sakmann B: Regulation of synaptic efficacy by coincidence of postsynaptic APs and EPSPs. Science 1997, 275(5297):2।3-2I5.

22. Wu LJ, Xu H, Ren M, Cao XY, Zhuo M: Parmacological Isolation of Postsynaptic Currents Mediated by NR2A- and NR2Bcontaining NMDA Receptors in the Anterior Cingulate Cortex. Molecular Pain 2007.

23. Rodrigues SM, Schafe GE, LeDoux JE: Intra-amygdala blockade of the NR2B subunit of the NMDA receptor disrupts the acquisition but not the expression of fear conditioning. J Neurosci 200I, 21 (I7):6889-6896.

24. Zhao MG, Toyoda H, Lee YS, Wu LJ, Ko SW, Zhang XH, Jia Y, Shum $\mathrm{F}, \mathrm{Xu} \mathrm{H}$, Li BM, Kaang BK, Zhuo M: Roles of NMDA NR2B subtype receptor in prefrontal long-term potentiation and contextual fear memory. Neuron 2005, 47(6):859-872.
25. Nakazawa T, Komai S, Watabe AM, Kiyama Y, Fukaya M, Arima-Yoshida F, Horai R, Sudo K, Ebine K, Delawary M, Goto J, Umemori H, Tezuka T, Iwakura Y, Watanabe M, Yamamoto T, Manabe T: NR2B tyrosine phosphorylation modulates fear learning as well as amygdaloid synaptic plasticity. Embo J 2006, 25(1 2):2867-2877.

26. Kohr G, Jensen V, Koester HJ, Mihaljevic AL, Utvik JK, Kvello A, Ottersen OP, Seeburg PH, Sprengel R, Hvalby O: Intracellular domains of NMDA receptor subtypes are determinants for long-term potentiation induction. J Neurosci 2003, 23(34): $1079 \mid-10799$.

27. Monyer H, Burnashev N, Laurie DJ, Sakmann B, Seeburg PH: Developmental and regional expression in the rat brain and functional properties of four NMDA receptors. Neuron 1994, I 2(3):529-540.

28. Barth AL, Malenka RC: NMDAR EPSC kinetics do not regulate the critical period for LTP at thalamocortical synapses. Nat Neurosci 200I, 4(3):235-236.

29. Sprengel R, Suchanek B, Amico C, Brusa R, Burnashev N, Rozov A Hvalby O, Jensen V, Paulsen O, Andersen P, Kim JJ, Thompson RF, Sun W, Webster LC, Grant SG, Eilers J, Konnerth A, Li J, McNamara JO, Seeburg PH: Importance of the intracellular domain of NR2 subunits for NMDA receptor function in vivo. Cell 1998, 92(2):279-289.

30. Bauer EP, Schafe GE, LeDoux JE: NMDA receptors and L-type voltage-gated calcium channels contribute to long-term potentiation and different components of fear memory formation in the lateral amygdala. J Neurosci 2002, 22(I 2):5239-5249.

31. Berberich SJV, Hvalby O, Seeburg PH, Kohr G: The role of NMDAR subtypes and charge transfer during hippocampal LTP induction. Neuropharmacology 2007, 52(I):77-86.

32. Hoffman DA, Sprengel R, Sakmann B: Molecular dissection of hippocampal theta-burst pairing potentiation. Proc Natl Acad Sci USA 2002, 99(I I):7740-7745.

33. Lisman J: Long-term potentiation: outstanding questions and attempted synthesis. Philos Trans R Soc Lond B Biol Sci 2003, 358( I 432):829-842.

34. Minichiello L, Calella AM, Medina DL, Bonhoeffer T, Klein R, Korte M: Mechanism of TrkB-mediated hippocampal long-term potentiation. Neuron 2002, 36(I):121-137.

35. Madison DV, Malenka RC, Nicoll RA: Mechanisms underlying long-term potentiation of synaptic transmission. Annu Rev Neurosci 1991, 14:379-397.

36. Lisman J, Schulman H, Cline H: The molecular basis of CaMKII function in synaptic and behavioural memory. Nat Rev Neurosci 2002, 3(3): $175-190$.

37. Gerkin RC, Lau PM, Nauen DW, Wang YT, Bi GQ: Modular competition driven by NMDA receptor subtypes in spike-timingdependent plasticity. J Neurophysiol 2007, 97(4):285 I-2862.

38. Zhou YD, Acker CD, Netoff TI, Sen K, White JA: Increasing Ca2+ transients by broadening postsynaptic action potentials enhances timing-dependent synaptic depression. Proc Natl Acad Sci USA 2005, I02(52): $19121-19125$.

39. Wei F, Wang GD, Kerchner GA, Kim SJ, Xu HM, Chen ZF, Zhuo M: Genetic enhancement of inflammatory pain by forebrain NR2B overexpression. Nat Neurosci 200I, 4(2): 164-169.

40. Wu LJ, Toyoda H, Zhao MG, Lee YS, Tang J, Ko SW, Jia YH, Shum FW, Zerbinatti CV, Bu G, Wei F, Xu TL, Muglia LJ, Chen ZF, Auberson YP, Kaang BK, Zhuo M: Upregulation of forebrain NMDA NR2B receptors contributes to behavioral sensitization after inflammation. J Neurosci 2005, 25(48): I I107-1 III6.

4I. Zhang XH, Liu F, Chen Q, Zhang CL, Zhuo M, Xiong ZQ, Li BM: Conditioning-strength dependent involvement of NMDA NR2B subtype receptor in the basolateral nucleus of amygdala in acquisition of auditory fear memory. Neuropharmacology 2008.

42. Priestley T, Laughton P, Myers J, Le Bourdelles B, Kerby J, Whiting P]: Pharmacological properties of recombinant human $\mathrm{N}$ methyl-D-aspartate receptors comprising NRIa/NR2A and NRIa/NR2B subunit assemblies expressed in permanently transfected mouse fibroblast cells. Mol Pharmacol 1995, 48(5):84I-848.

43. Carmignoto G, Vicini S: Activity-dependent decrease in NMDA receptor responses during development of the visual cortex. Science 1992, 258(5084): |007-101|. 
44. Sans N, Petralia RS, Wang YX, Blahos f 2nd, Hell JW, Wenthold RJ: A developmental change in NMDA receptor-associated proteins at hippocampal synapses. I Neurosci 2000, 20(3): |260-|27|.

45. Barria A, Malinow R: NMDA receptor subunit composition controls synaptic plasticity by regulating binding to CaMKII. Neuron 2005, 48(2):289-30I.

46. Husi H, Ward MA, Choudhary JS, Blackstock WP, Grant SG: Proteomic analysis of NMDA receptor-adhesion protein signaling complexes. Nat Neurosci 2000, 3(7):66I-669.

47. Steigerwald F, Schulz TW, Schenker LT, Kennedy MB, Seeburg PH, Kohr G: C-Terminal truncation of NR2A subunits impairs synaptic but not extrasynaptic localization of NMDA receptors. J Neurosci 2000, 20(I 2):4573-458I.

48. Vissel B, Krupp J], Heinemann SF, Westbrook GL: Intracellular domains of NR2 alter calcium-dependent inactivation of $\mathbf{N}$ methyl-D-aspartate receptors. Mol Pharmacol 2002, 6I(3):595-605.

49. Frizelle PA, Chen PE, Wyllie DJ: Equilibrium constants for (R)[(S)-I-(4-bromo-phenyl)-ethylamino]-(2,3-dioxo-I,2,3,4-tetrahydroquino xalin-5-yl)-methyl]-phosphonic acid (NVPAAM077) acting at recombinant NRI/NR2A and NRI/NR2B $\mathrm{N}$-methyl-D-aspartate receptors: Implications for studies of synaptic transmission. Mol Pharmacol 2006, 70(3): 1022-1032.

50. Paoletti $P$, Neyton J: NMDA receptor subunits: function and pharmacology. Curr Opin Pharmacol 2007, 7(I):39-47.

Publish with Bio Med Central and every scientist can read your work free of charge

"BioMed Central will be the most significant development for disseminating the results of biomedical research in our lifetime. "

Sir Paul Nurse, Cancer Research UK

Your research papers will be:

- available free of charge to the entire biomedical community

- peer reviewed and published immediately upon acceptance

- cited in PubMed and archived on PubMed Central

- yours - you keep the copyright
BioMedcentral 